\title{
Complement susceptibility in glutamine deprived breast cancer
} \section{cells}

\author{
Bradley S Ellison* ${ }^{* 1}$, Mary KB Zanin ${ }^{\dagger 2}$ and Robert J Boackle ${ }^{\dagger 3}$
}

Address: ${ }^{1}$ Department of Orthopaedic Surgery, The Ohio State University, 410W 10th Ave, Columbus Ohio 43210, USA, ${ }^{2}$ Department of Biology, The Citadel, 171 Moultrie Street, Charleston, SC 29409, USA and ${ }^{3}$ Department of Stomatology and Department of Immunology, Medical University of South Carolina, 171 Ashley Ave., Charleston, South Carolina 29425, USA

Email: Bradley S Ellison* - Brad.Ellison@osumc.edu; Mary KB Zanin - zaninm1@citadel.edu; Robert J Boackle - boacklrj@musc.edu

* Corresponding author †Equal contributors

Published: II July 2007

Cell Division 2007, 2:20 doi:10.1186/1747-1028-2-20

This article is available from: http://www.celldiv.com/content/2/l/20

(c) 2007 Ellison et al; licensee BioMed Central Ltd.

This is an Open Access article distributed under the terms of the Creative Commons Attribution License (http://creativecommons.org/licenses/by/2.0), which permits unrestricted use, distribution, and reproduction in any medium, provided the original work is properly cited.
Received: 16 November 2006

Accepted: II July 2007

\begin{abstract}
Background: Membrane complement regulatory proteins (mCRPs) inhibit complement-mediated killing of human cells by human complement, a property that confers protection from complement to malignant breast cancer cells and that thwarts some immunotherapies. Metabolic mechanisms may come into play in protecting cancer cells from the complement system subsequent to relatively low levels of complement deposition.
\end{abstract}

Results: In differentiating these mechanisms, two types of human breast cancer cell lines, MCF7 (adenocarcinoma) and Bcap37 (medullary carcinoma) were cell-cycle synchronized using glutamine-deprivation followed by restoration. These cells were examined for the expression of two mCRPs (CD59 and CD55), and for subsequent susceptibility to antibody-mediated complement-induced membrane damage. After glutamine restoration, MCF7 and Bcap37 cells were synchronized into the G2/M phase and an average increased expression of CD59 and CD55 occurred with a corresponding resistance to complement-mediated damage. Blocking CD59 inhibitory function with monoclonal antibody revealed that CD59 played a key role in protecting unsynchronized Bcap37 and MCF7 cancer cells from the complement membrane attack complex. Interestingly, glutamine-deprivation did not significantly affect the expression of proteins e.g., the surface level of CD59 or CD55, but did increase the susceptibility to complement-mediated killing. One possible explanation is that glutamine-deprivation may have slowed the turnover rate of mCRPs, preventing the cells from replacing pre-existing mCRPs, as they became neutralized by covalent $\mathrm{C} 4 \mathrm{~b}$ and $\mathrm{C} 3 \mathrm{~b}$ depositions.

Conclusion: Taken together the findings are consistent with the conclusion that future immunotherapies should aim to achieve a highly specific and profound activation and deposition of complement as well as to disrupt the synthesis and expression of CD59 and CD55 by the cancer cells.

\section{Background}

The complement system is a critical arm of the immune system that allows the system to eliminate pathogens.
Complement is classically activated by the presence of antibodies on cell surface antigens. If allowed to proceed uninhibited, a complement activation cascade occurs that 
culminates in elimination of an antibody-coated cell via phagocytosis or lysis. Complement control mechanisms maximize the efficiency of the complement system in specifically targeting sensitized pathogens, while sparing incidental damage to nearby host cells. Indeed, membraneassociated complement regulatory proteins (mCRPs) are often over-expressed on host cells in areas of inflammation and restrict complement-mediated membrane damage after the inadvertent deposition of the complement components $\mathrm{C} 4 \mathrm{~b}$ or $\mathrm{C} 3 \mathrm{~b}$ [1]. In addition, malignant cancer cells express mCRPs $[2,3]$ and may release soluble forms of selected mCRPs [4] which protect them against complement-mediated cell killing.

Fluid phase complement regulators, such as $\mathrm{C} 1$-inhibitor prevent the unnecessary consumption and depletion of soluble complement components, so as to allow a more effective and specifically directed complement-mediated attack on sensitized pathogens. However in the absence of sufficient levels of antibody deposition, this and other complement control mechanisms tend to restrict the ability of complement to eliminate cancer cells [2,5-9]. Current therapeutic mAbs as well as endogenous low affinity IgG antibodies to cancer cells often recruit the comple-

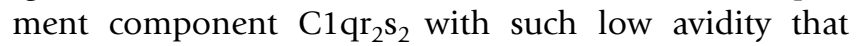
serum C1-inhibitor is able to rapidly inhibit activated C1r and $\mathrm{C} 1 \mathrm{~s}$, and in most cases quickly remove the entire

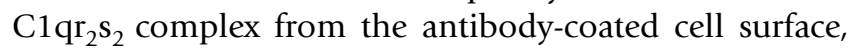
resulting in only a trace level of C4b-deposition [10-12]. Meanwhile, CD55 and CD46 on malignant cells restrict deposited C4b and C3b, and CD59 inhibits complement membrane attack complex formation, therein protecting cancer cells from membrane damage $[13,14]$. When the complement cascade is strongly activated, the complement components C4b and C3b bind to mCRPs and inactivate them, but low levels of complement depositions are incapable of neutralizing sufficient percentages of the expressed mCRPs on the surface of the cancer cells. Indeed, repeated low level, albeit ineffective, complement-depositions are capable of inducing metabolic consequences that may result in an undesirable increase in resistance to apoptotic influences $[15,16]$ and could enhance resistance to complement-mediated killing $[17,18]$, therein providing an acquired advantage for the surviving cancer cell populations [19]. Therefore, when designing antibody therapies to utilize complement in the elimination of malignant cancer cells, highly effective classical pathway activation may be needed to mediate sufficient C4b and C3b depositions to covalently bind and block the function of mCRPs on the targeted cancer cell surface.

Cell cycle dependent phenomena and resultant expression of targeted antigens have been associated with variant susceptibilities to complement-mediated lysis [20-
22]. However, many of these studies were conducted just as CD59 and CD55 were being fully characterized and several studies used cross-species complement. In addition, few reports have examined the effect of cell-cycle synchronization on the expression of mCRPs in breast cancer cell lines. In this study, the expression levels of CD59 and CD55 were measured in two different types of human breast cancer cell lines, a human breast adenocarcinoma cell line MCF7 [23] and a human breast medullary carcinoma cell line Bcap37 [24] before and after cell cycle synchronization using glutamine-deprivation and restoration. The expression levels of CD59 and CD55 were correlated to susceptibility to human complementmediated lysis following complement activation. Complement was activated by exposing cancer cells to excess levels of polyclonal rabbit antibodies to $\beta_{2}$-microglobulin, a stably and abundantly expressed antigen on cancer cell line surfaces $[23,25]$. Use of this polyclonal antibody to activate $\mathrm{C} 1$ blocks the rapid entrance and action of $\mathrm{C} 1$ inhibitor $[10,11]$ allowing sufficient progression of the complement cascade so as to allow a relative measurement of the impact of mCRPs on complement-mediated cell damage.

In these studies we found that glutamine deprivation increases breast cancer cell susceptibility to complementmediated killing. We also found that subsequent glutamine restoration produces cancer cells that substantially up-regulate the expression of the mCRPs CD59 and CD55 on the cell surface and that these glutaminerestored cancer cells are even more resistant to complement-mediated damage than unsynchronized breast cancer cells.

\section{Results \\ Complement-mediated lysis of antibody-coated breast cancer cells as a function of the level of normal human serum as a source of complement}

Normal human serum (NHS) is a readily available and reliable source that contains the entire battery of complement proteins needed to evoke the immunological cascade of antibody-directed complement-mediated cell lysis. As lytic damage to the cell membrane occurs, intracellular enzymes and elements are released into the extracellular domain, allowing them to become markers in determining the extent of cellular damage incurred during complement-mediated lysis. In this manner, a commercially available Lactate Dehydrogenase (LDH) assay (Sigma Diagnostics) was employed to detect the extent of total complement-mediated cell lysis.

In NHS, however, various levels of background LDH activity can be detected. Serial dilutions of NHS were developed to identify the most appropriate concentration of NHS that could be used in further experimentation dem- 
onstrating minimal background LDH activity, while optimizing detection of LDH activity as an index of antibodydirected complement-mediated lysis. II BothThe use of $25 \%$ NHS as a source of complement provided the maximal level of complement-mediated lysis [26], while allowing an acceptable serum-LDH background. Detection of complement-mediated membrane damage to anti$\beta_{2}$-microglobulin sensitized cancer cells, monitored via release of LDH (and Trypan Blue exclusion), indicated that $20 \%$ of the MCF7 cells and $18 \%$ of the Bcap37 cells were lysed (Figure 1). As NHS was further diluted, both MCF7 and Bcap37 cells became increasingly resistant to complement-mediated membrane damage. Therefore $25 \%$ NHS as a source of complement was used throughout the remaining studies.

\section{Neutralization of CD59 enhanced complement-mediated lysis of breast cancer cells}

The cell surface marker CD59 (Protectin) prevents formation of the membrane attack complex, the terminal arm of the activated complement system responsible for inducing cell lysis. Thus, CD59 is considered one of the most important innate defense mechanisms cells have against complement activation. The YTH53.1 monoclonal antibody (Serotec, Raleigh, NC) has been proven to bind to CD59 on the surface of human cells and neutralize the complement inhibitory function of CD59 $(37,38)$. Our objective was to determine the specific degree of protection CD59 offers MCF7 and Bcap37 breast cancer cells from antibody-directed complement-mediated damage.

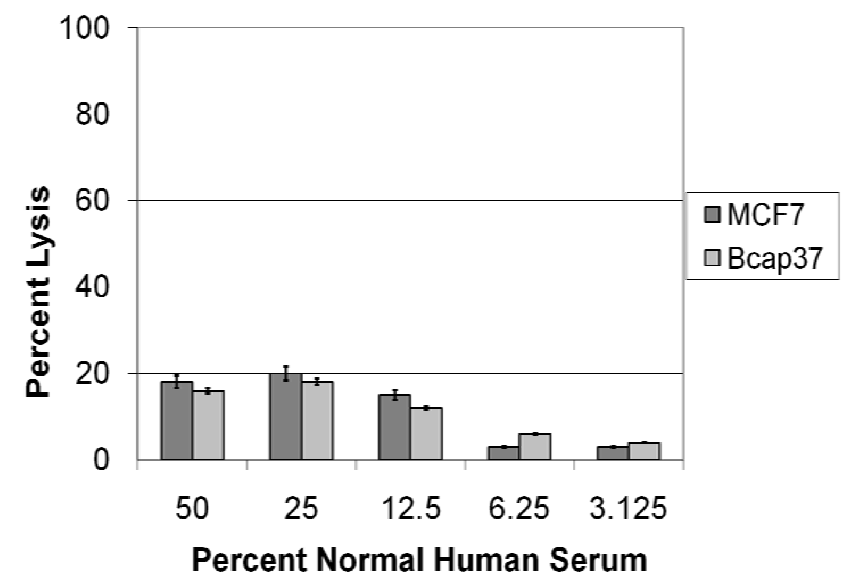

\section{Figure I}

Optimal NHS Dilution for Complement-mediated lysis of human breast cancer cell lines. The Bcap37 and MCF7 cells (I $\times 10^{5}$ ) were sensitized $20 \mu \mathrm{g}$ rabbit antibody to $\beta_{2^{-}}$ microglobulin and incubated with specified dilutions of normal human serum (NHS). After a total 4.5 hours of incubation at $37^{\circ} \mathrm{C}$, supernatants were collected and tested for $\mathrm{LDH}$ activity to determine percent lysis.
The MCF7 and Bcap37 cells received the combined treatment of YTH53.1 along with anti- $\beta_{2}$-microglobulin antibodies followed by $25 \%$ NHS. Cells treated with only the YTH53.1 anti-CD59 monoclonal antibody demonstrated only $7 \%$ lysis for Bcap37 and 11\% lysis for MCF7 cells (Figure 2, Treatment group 3). The relatively small levels of lysis attributed to YTH53.1 alone were due to inhibition of CD59 function and mild complement activation and regarded as background lysis. When treated with only $20 \mu \mathrm{g}$ of rabbit polyclonal anti- $\beta_{2}$-microglobulin antibodies, the percent lysis for Bcap37 cells was 33\% and for MCF7 cells was $42 \%$. However, when $20 \mu \mathrm{g}$ of YTH53.1 anti-CD59 monoclonal antibodies were combined with the anti- $\beta_{2}$-microglobulin antibodies, the percent lysis significantly increased to $52 \%$ for Bcap37 cells (Figure 2, Treatment groups B and F: p value $=0.009)$ and $59 \%$ for MCF7 cells (Figure 2, Treatment groups B and F: $p$ value $=$ 0.005). Thus, neutralization of CD59 function with YTH53.1 significantly increased complement-mediated lysis of breast cancer cells.

No significant difference was observed between Bcap37 and MCF7 cells treated with $20 \mu \mathrm{g}$ of YTH53.1 and either 10 or $20 \mu \mathrm{g}$ of rabbit polyclonal anti- $\beta_{2}$-microglobulin

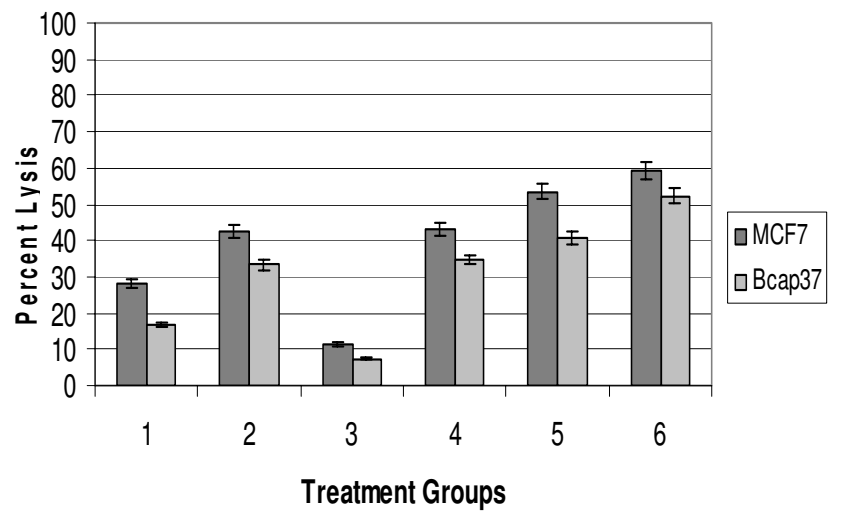

Figure 2

Neutralization of CD59 enhanced complement-mediated lysis. In A through D, Bcap37 and MCF7 cells $\left(1 \times 10^{5}\right)$ were sensitized with $20 \mu \mathrm{g}$ of rabbit antibody to $\beta_{2}$-microglobulin (anti- $\beta 2 M$ ) with or without specified doses of $\mathrm{mAb}$-YTH53.I (anti-CD59). Background LDH activity was obtained with unsensitized cells similarly incubated with $25 \%$ NHS for 4.5 hours to provide a numerical value that was subtracted from experimental values. Experimental antibody combinations are represented as follows: I) $10 \mu \mathrm{g}$ anti- $\beta 2 \mathrm{M}$ alone $(28.0 \%$ lysis \pm I.7). 2) $20 \mu \mathrm{g}$ anti- $\beta 2 \mathrm{M}$ alone (42.3\% lysis \pm 2.9$)$. 3) $20 \mu \mathrm{g}$ mAb-YTH53.I alone (I I.3\% lysis \pm I.5). 4) $20 \mu \mathrm{g}$ anti- $\beta 2 \mathrm{M}+$ $5 \mu \mathrm{g}$ mAb-YTH53.I (43.3\% lysis \pm 2.1$)$. 5) $20 \mu \mathrm{g}$ anti- $\beta 2 M+$ $10 \mu \mathrm{g} \mathrm{mAb-YTH53.I} \mathrm{(53 \%} \mathrm{lysis} \pm 4.8)$. 6) $20 \mu \mathrm{g}$ anti- $\beta 2 M+$ $20 \mu \mathrm{g}$ mAb-YTH53.I (59\% lysis \pm 4.9$)$. 
antibodies. However, Bcap37 cells treated with anti- $\beta_{2}-$ microglobulin antibodies and $5 \mu \mathrm{g}$ of YTH53.1 demonstrated $34 \%$ lysis, while cells treated with anti- $\beta_{2^{-}}$ microglobulin antibodies and $20 \mu \mathrm{g}$ of YTH53.1 demonstrated 52\% lysis (Figure 2, Treatment groups D and F; p value $=0.05)$. Likewise, MCF7 cells treated with anti- $\beta_{2}-$ microglobulin antibodies and $5 \mu \mathrm{g}$ of YTH53.1 demonstrated $43 \%$ lysis, while cells treated with anti- $\beta_{2}-$ microglobulin antibodies and $20 \mu \mathrm{g}$ of YTH53.1 demonstrated 59\% lysis (Figure 2, Treatment groups D and F; p value $=0.02$ ). With the YTH53.1 antibody demonstrating a dose-response, the $20 \mu \mathrm{g}$ of YTH53.1 was selected as an effective amount in eliciting complement-mediated lysis for further experimentation.

\section{Cell cycle synchronization with glutamine deprivation and restoration}

Using flow cytometry, cells in different phases of the cell cycle are identified based on the amount of DNA present inside the cell, since duplication of genetic material occurs during progression of the cell cycle when a diploid cell prepares for mitotic division. Specifically, propidium iodide intercalates within DNA providing a fluorescent marker that allows measurement of total DNA material by flow cytometry. Diploid cells in the G0-G1 phase are denoted as having a total DNA content of $2 \mathrm{C}$, while cells in $S$ phase have a total DNA content between $2 \mathrm{C}$ and $4 \mathrm{C}$, and cells in G2-M phase have a total DNA content of 4C. Discrimination between G0 and G1 phases is not possible because the amount of DNA is identical (2C) for cells in either the G0 or G1 phase, thus cells with 2C DNA were grouped together as G0-G1 phase. Similarly, the amount of DNA is identical for cells in either the G2 or M phase, thus cells with 4C DNA were grouped together as G2-M phase.

Glutamine is an amino acid routinely added to cell culture media due to its importance in the regulation of cell metabolism through its association with the synthesis of both protein and DNA (27). Glutamine-deprivation has been used as a method of arresting cells in the G0 phase of the cell cycle (39). When glutamine is restored to the culture media, the cells are able to then proceed unimpeded through the cell cycle. After Bcap37 and MCF7 cells had been deprived of glutamine for 48 hours, $2 \mathrm{mM} \mathrm{L}$ glutamine was added to the culture medium. Distinct cell cycle profiles were characterized for Bcap37 and MCF7 cells subjected to glutamine synchronization when compared with unsynchronized cells. Distribution of population averages were calculated for G0-G1 phase, S phase, and G2-M phase among unsynchronized and synchronized cells. Unsynchronized Bcap37 cells demonstrated $61 \%$ in G0-G1 phase, $22 \%$ in S phase and $16 \%$ in G2-M phase (figure 3a). Glutamine deprived Bcap37 cells demonstrated $61 \%$ in G0-G1 phase, $32 \%$ in $S$ phase and $4 \%$ in G2-M phase. Glutamine restored Bcap37 cells demonstrated 25\% in G0-G1 phase, 34\% in S phase and $41 \%$ in G2-M phase. Unsynchronized MCF7 cells demonstrated $60 \%$ in G0-G1 phase, $24 \%$ in S phase and $17 \%$ in G2-M phase (figure 3a). Glutamine deprived MCF7 cells demonstrated $68 \%$ in G0-G1 phase, $24 \%$ in $S$ phase and $4 \%$ in G2-M phase. Glutamine restored MCF7 cells demonstrated $27 \%$ in G0-G1 phase, 35\% in S phase and 38\% in G2-M phase. Thus, the cell cycle profile changes significantly depending on the presence or absence of glutamine revealing that glutamine depletion synchronizes cells by arresting progression predominantly in G0-G1 phase. However, upon restoration of glutamine the cell cycle profile is further altered with an abrupt forward progression into G2-M phase. Therefore, glutamine depletion and restoration affords the unique ability to synchronize the cell cycle, resulting in significant shifts in the proportion of MCF7 and Bcap37 cells in G0-G1 phase, S phase or G2-M phase. Further, cells receiving glutamine following glutamine deprivation demonstrated a common shift in the proportion of cells in the quiescent G0-G1 phase to active mitotic proliferation of G2-M phase, the most characteristic stage of neoplastic transformation.

\section{The effect of glutamine deprivation and restoration on the expression of CD59 and CD55}

With glutamine synchronization resulting in significant shifts in the cell cycle profiles for Bcap37 and MCF7 cells, the cells were then examined to determine if glutamine synchronization impacted CD59 and CD55 expression. Bcap37 and MCF7 cells were synchronized with glutamine deprivation for 48 hours, then subsequently received $2 \mathrm{mM}$ L-glutamine for 8 hours. Following glutamine restoration, cells were harvested and prepared analysis by flow cytometry. Using the ModFit LT software, the Mean Fluorescence Intensity (MFI) was examined with the cell acquisition being approximately 5,000 cells for each treatment group (data not shown). The MFI was examined for CD59, CD55 and the appropriate negative isotype controls for unsynchronized and glutamine restored cells. The negative background isotype controls (non-specific FITC-labeled mouse immunoglobulins, IgG2a and $I g G$ ) revealed negligible cell surface binding. Compared with unsynchronized cells, glutamine restoration resulted in a 3.5-fold increase in and a 3.1-fold increase in CD55 expression for Bcap37 cells (Figure 4a). Similar results were observed for MCF7 cells upon glutamine-restorationwith a 3.7-fold increase in CD59 expression and a 2.2-fold increase in CD55 expression (Figure 4b). Thus, glutamine restoration resulted in increased CD59 and CD55 expression for Bcap37 and MCF7 cells when controlled for cell number. Therefore, glutamine restoration not only induced Bcap37 and MCF7 cells to rapidly progress through the cell cycle, but also augmented the 


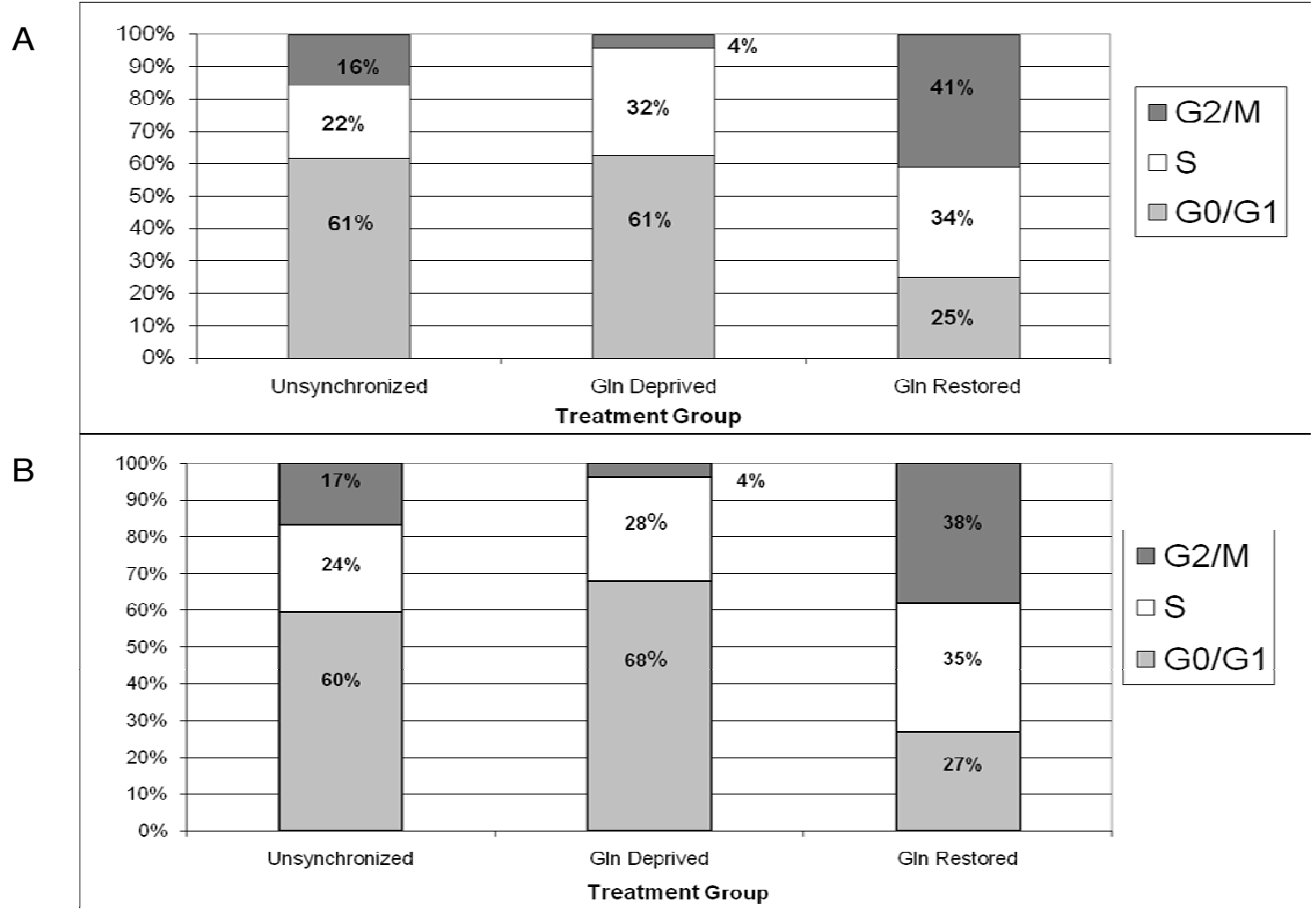

\section{Figure 3}

Cell cycle distribution for breast cancer cells subjected to glutamine-mediated synchronization. Data are depicted as representative means for three independent experiments. Bcap37 cells (Figure 3a) and MCF7 cells (Figure 3b) were grown to confluency and either maintained in media with glutamine (Unsynchronized) or without glutamine for 48 hours (Gln Deprived), or without glutamine for 48 hours but then followed by 8 hours with glutamine (Gln Restored). All cells were harvested and treated with $0.5 \mathrm{mg} / \mathrm{ml}$ propidium iodide to determine cell cycle distribution by FACS analyses.

levels of CD59 and CD55 expression along with the mitotic index.

\section{Comparison of CD59 and CD55 expression in G0-GI and G2-M phases of the cell cycle}

Given the distinct cell cycle profiles observed with glutamine-deprived and glutamine-restored cells, the possibility existed that the increase in CD59 and CD55 expression might be directly related to the cell cycle phase. To determine if CD59 or CD55 expression was phase specific, flow cytometry was used to isolate the G0-G1 and the G2-M subpopulations of Bcap37 and MCF7 cells within each synchronization group. During Cell Analysis using the FACSCalibur device and ModFit LT software, the distinct populations of cells were identified in either G0G1 or G2-M based on channel values dictated by the amount of propidium iodide intercalated within DNA. All diploid cells (2C) were identified, then separated according to channel values based on propidium iodide fluores- cence at $585 \mathrm{~nm}$ (data not shown). Cells identified as G0G1 had channel values ranging approximately from 25 to 75. Cells identified as G2-M had channel values ranging approximately from 140 to 175 . Gating was performed manually after identification of G0-1 and G2-M populations to further determine CD59 and CD55 MFI among these distinct populations of cells at different stages in the cell cycle. Using the ModFit LT software, the Mean Fluorescence Intensity (MFI) was examined with the cell acquisition being approximately 5,000 cells for each treatment group (data not shown). The MFI was examined for CD59, CD55 and the appropriate negative isotype controls in the G0-G1 and G2-M channel ranges for unsynchronized and glutamine restored cells. The negative background isotype controls (non-specific FITC-labeled mouse immunoglobulins, IgG2 $a$ and IgG) revealed negligible cell surface binding. Since glutamine-deprived Bcap37 and MCF7 cells had only $4 \%$ of total cells in G2-M, an inadequate number of cells were available for determina- 


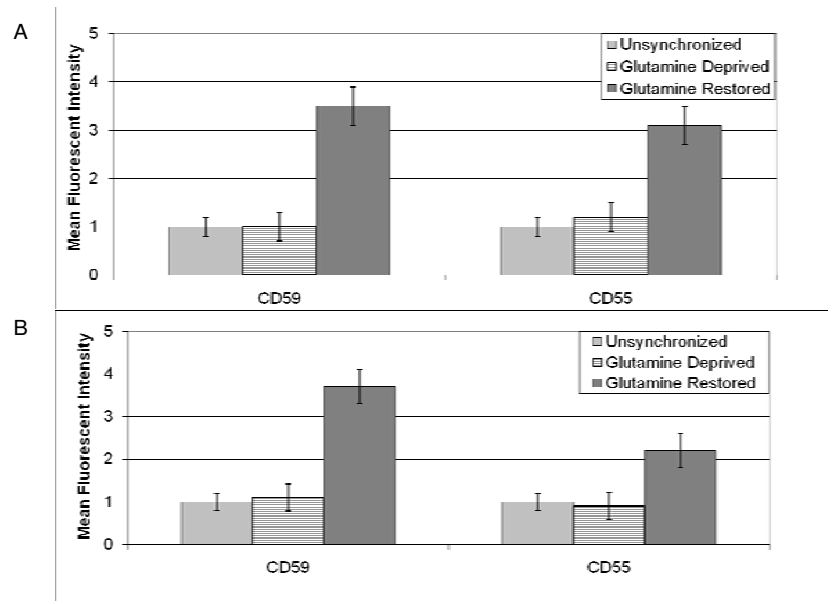

\section{Figure 4}

Expression of CD59 and CD55 in breast cancer cells subjected to glutamine-mediated synchronization. Data is depicted as representative means for three independent experiments. Bcap37 cells (Figure 4a) and MCF7 cells (Figure $4 \mathrm{~b})$ were grown to confluency and either maintained with glutamine (Unsynchronized) or in the same media without glutamine for 48 hours (Glutamine Deprived), or in the same media without glutamine for 48 hours followed by 8 hours with glutamine (Glutamine Restored). In all experimental groups for Bcap37 and MCF7 cells, CD59 was quantified using FITC-conjugated anti-CD59 mouse monoclonal antibody and CD55 was quantified using FITC-conjugated antiCD55 mouse monoclonal antibody.

tion of MFI for CD59 and CD55. However, the G2-M subpopulations for the unsynchronized and glutamine restored Bcap37 and MCF7 cells contained a sufficient number of cells for analysis. When comparing the MFI for CD59 and CD55 between G0-G1 and G2-M subpopulations of cells in different phases of the cell cycle, no significant difference in mCRP expression was observed for Bcap37 or MCF7 cells (Figures 5a and 5b). Therefore, the increased expression of CD59 and CD55 observed with glutamine restoration appears to not be directly related to the increased proportion of cells in the G2-M phase of the cell cycle. Rather, other factors that are not cell cycledependent, yet associated with the metabolic proliferation stimulated by glutamine restoration are responsible for the increased expression of mCRPs.

\section{Influence of glutamine deprivation or restoration on complement susceptibility}

With the presence or absence of glutamine impacting the expression levels of mCRPs, the susceptibility of breast cancer cells to complement-mediated lysis also is likely dependent on the relative level of glutamine in the cellu- lar microenvironment. To determine if changes in expression levels of CD59 and CD55 altered their susceptibility to complement-mediated lysis, unsynchronized, glutamine-deprived and glutamine-restored cells were treated with complement-activating antibodies and incubated with NHS for 4 hours to determine the percentage of complement-mediated lysis. When treated with $20 \mu \mathrm{g}$ of rabbit polyclonal anti- $\beta_{2}$-microglobulin antibodies, unsynchronized Bcap37 cells demonstrated 26\% lysis (Figure 6). However, when Bcap37 cells were cultured in a glutamine-deficient medium, complement-mediated damage significantly increased to $37 \%$ ( $p$ value $=0.007$ ). Further, when glutamine was restored to the media following a 48-hour period of deprivation, Bcap37 cells demonstrated $17 \%$ lysis, a significant reduction in complement susceptibility compared to either unsynchronized or glutamine deprived cells ( $\mathrm{p}$ values $=0.05$ and 0.003 , respectively). The impact of glutamine synchronization affected the MCF7 cells similarly. When treated with $20 \mu \mathrm{g}$ of rabbit polyclonal anti- $\beta_{2}$-microglobulin antibodies, unsynchronized MCF7 cells demonstrated $37 \%$ lysis. Yet, MCF7 cells cultured in glutamine-deficient media demonstrated a significant increase in complement susceptibility with $54 \%$ lysis ( $\mathrm{p}$ value $=0.01$ ). Additionally, when glutamine is restored to the media, MCF7 cells demonstrated a significant decrease in complement susceptibility compared with glutamine-deprived cells illustrated by $26 \%$ lysis ( $\mathrm{p}$ value $=0.007$ ).

As expected, glutamine restored Bcap37 and MCF7 cells with increased CD59 and CD55 expression exhibited heightened resistance to complement-mediated lysis. Interestingly, when compared with unsynchronized cells, glutamine deprived Bcap37 and MCF7 cells demonstrated increased susceptibility to complement-mediated lysis although expression levels of CD59 and CD55 were similar (figures $4 \mathrm{a}$ and $4 \mathrm{~b}$ ). Thus, the increased expression of CD59 and CD55 enhance complement resistance, however, other factors associated with glutamine deprivation beyond expression levels of mCRPs also influence complement susceptibility.

\section{Neutralization of CD59 augments complement-mediated lysis following glutamine synchronization}

Glutamine synchronization altered the expression levels of mCRPs, cell cycle profiles, and complement susceptibility among breast cancer cells, suggesting microenvironmental perturbations likely influence complementactivating antibody-directed breast cancer therapies.

One of the most effective mCRPs is CD59, which functions to prevent formation of the membrane attack complex specifically blocking the terminal arm of the complement cascade. The YTH53.1 monoclonal antibody (Serotec, Raleigh, NC) has been proven to bind to CD59 


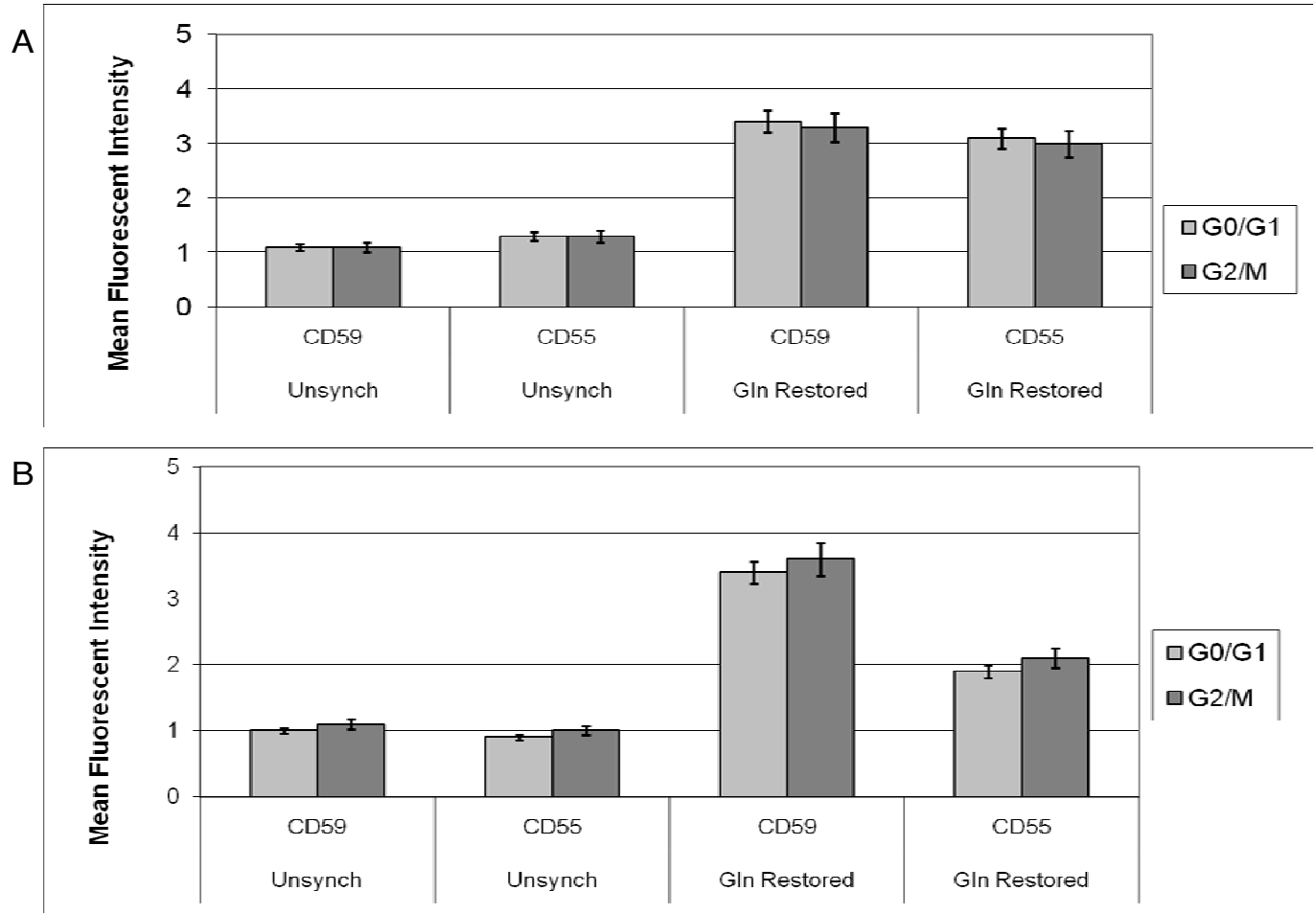

\section{Figure 5}

CD59 and CD55 expression in G0-GI and G2-M subpopulations. Data is depicted as representative means for three independent experiments. Unsynchronized Bcap37 cells (Figure 5a) and MCF7 cells (Figure 5b) were grown to confluency and maintained in media supplemented with $2 \mathrm{mM} \mathrm{L-glutamine} \mathrm{(Unsynch).} \mathrm{Synchronized} \mathrm{cells} \mathrm{were} \mathrm{grown} \mathrm{to} \mathrm{confluency} \mathrm{in} \mathrm{normal}$ media and then maintained in media without glutamine for 48 hours, followed by 8 hours in media supplemented with 2 mM Lglutamine (Gln Restored). Cells were then harvested, treated with propidium iodide, incubated with either FITC-conjugated antibodies to CD59 or to CD55 and prepared for flow cytometric sorting and analysis.

on the surface of human cells and neutralize its complement inhibitory function $(37,38)$. Our objective was to determine the specific degree of protection CD59 offers Bcap37 and MCF7 breast cancer cells from antibodydirected complement-mediated damage when simultaneously challenged with glutamine synchronization.

For Bcap37 and MCF7 cells, the YTH53.1 monoclonal antibody was used in combination with complement activating anti- $\beta_{2}$-microglobulin antibodies and $25 \%$ NHS. Unsynchronized Bcap37 cells demonstrated 42\% lysis (Figure 7). Following glutamine deprivation, Bcap37 cells revealed a significant increase in complement-mediated lysis of $61 \%$ ( $p$ value $=0.05$ ). Additionally, glutamine restoration resulted in 31\% lysis, revealing decreased complement susceptibility when compared with the unsynchronized and glutamine-deprived treatment groups ( $\mathrm{p}$ values $=0.02$ and 0.01 , respectively). (All of these lysis values for Bcap37 cells are higher than those of cells treated with only anti- $\beta_{2}$-microglobulin as seen in Figure 6, where unsynchronized Bcap37 cells demonstrated 26\% lysis, glutamine deprived Bcap37 cells demonstrated 37\% lysis, and glutamine restored Bcap37 cells demonstrated $17 \%$ lysis). Similar observations were seen with MCF7 cells, with unsynchronized cells demonstrating 55\% lysis. Following glutamine deprivation, MCF7 cells showed a significant increase in complement-mediated lysis of $72 \%$ ( $\mathrm{p}$ value $=0.001)$. Further, glutamine restoration resulted in decreased complement susceptibility with $46 \%$ lysis, a significant decrease compared with unsynchronized and glutamine-deprived MCF7 cells ( $p$ value $=0.02$ and 0.01 , respectively). (All of these lysis values for MCF7 cells are higher than those of cells treated with only anti- $\beta_{2}$-microglobulin as seen in Figure 6 , where unsynchronized MCF7 cells demonstrated 37\% lysis, glutamine deprived MCF7 cells demonstrated 54\% lysis, and glutamine restored MCF7 cells demonstrated 26\% lysis). 


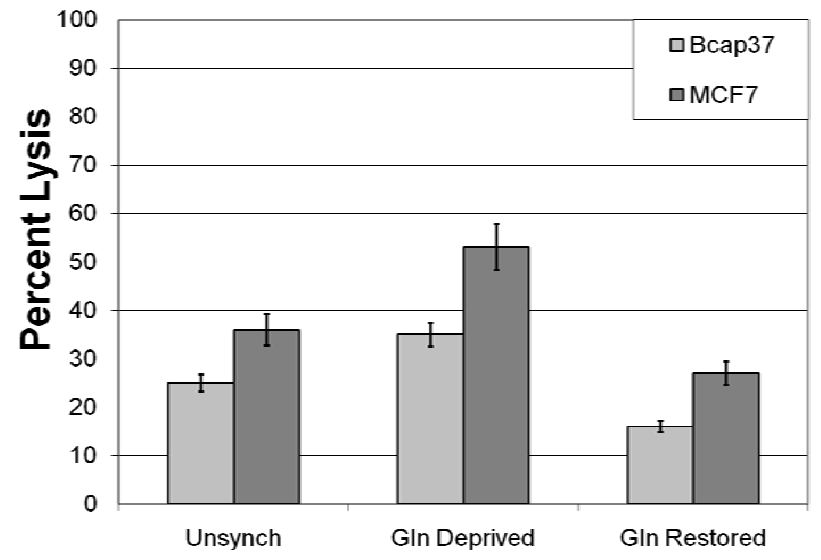

Figure 6

The effect of glutamine-mediated synchronization on complement susceptibility in breast cancer cell lines. All populations of cells were grown to confluency in separate culture flasks. Unsynchronized cells were maintained in 10\% FBS DMEM supplemented with $2 \mathrm{mM}$ L-glutamine, while some cells were subsequently maintained in glutamine deficientmedia for 48 hours (GIn Deprived), and of this group, some were subsequently supplemented with $2 \mathrm{mM}$ L-glutamine for 8 hours (Gln Restored). All populations of cells were sensitized with $20 \mu \mathrm{g}$ of rabbit polyclonal antibody to $\beta_{2^{-}}$ microglobulin and subjected to $25 \%$ fresh normal human serum (NHS). After 4.5 hours of incubation with NHS at $37^{\circ} \mathrm{C}$, supernatants were collected and tested for LDH activity to determine percent lysis.

In view of these results, CD59 offers significant resistance to complement-mediated lysis regardless of whether cells are subjected to glutamine synchronization or not. However, when Bcap37 and MCF7 cells are faced with potent complement activation in the face of glutamine deprivation and CD59 neutralization, complement susceptibility is markedly increased. Conversely, restoration of glutamine seemingly reverses the increased complement susceptibility observed with glutamine deprivation. However, neutralization of CD59 combined with potent complement activation still elicited appreciable complementmediated lysis when compared to glutamine-restored cells not treated with YTH53.1 (Figure 6). Additionally, glutamine-restored Bcap37 and MCF7 cells exhibited increased complement resistance than their unsynchronized counterparts, even when CD59 was neutralized for both treatment groups. Therefore, glutamine restoration not only increases complement resistance by elevating CD59 expression, but also likely induced other yet unidentified intracellular responses that provided additional resistance to complement-mediated lysis.

\section{Discussion}

Membrane complement regulatory proteins (mCRPs) that are expressed on breast cancer cells, as well as normal cells provide exquisite protection from complement-mediated lysis [13,28-30], after inadvertent $\mathrm{C} 4 \mathrm{~b}$ or $\mathrm{C} 3 \mathrm{~b}$ deposition or after a marginal initiation of the classical pathway subsequent to sensitization of cancer cells by exogenous monoclonal antibodies or low affinity endogenous antibodies. Should the complement cascade override the effects of CD55 (and CD46, another mCRP) and manage to activate the terminal complement components on cancer cell membranes, functional CD59 molecules bind to human $\mathrm{C} 8$ and $\mathrm{C} 9$, during the attempt to properly assemble the membrane attack complex [31]. By preventing complement-mediated membrane damage, CD59 conveys an immunological privilege to both normal host and neoplastic cells $[1,5,6,32]$. The soluble complement inhibitor, C1-inhibitor, acts at the sensitized surface to remove $\mathrm{C} 1$ and block the classical pathway, but a highly effective initiation of the classical complement pathway by abundant levels of complement-activating polyclonal antibodies to numerous proximate antigenic determinants on cancer cell surfaces may override C1-inhibitor [10-12], and, as shown here, may generate a profound fixation of complement that results in a significant percentage of cell killing as measured by the release of LDH and uptake of Trypan Blue.

In these experiments, serum is the source of complement, but the serum also contains $\mathrm{C} 1$-inhibitor. The dilution of serum from $50 \%$ to $25 \%$ caused an increased complement-mediated lysis of the sensitized cancer cells (Figure 1 ). We have reported previously that $\mathrm{C} 1$-inhibitor function is the rate-limiting step in the initiation of the classical pathway $[10,11]$. Therefore we suggest that under the conditions of our experiment, the level of functional C1inhibitor was reduced to a defined point that allowed an increase in C1 deposition, yet did not significantly compromise C1-inhibitor's ability to control fluid-phase C1 activation, which would otherwise result in depletion of soluble complement components.

It may seem unlikely that cells expressing significant levels of mCRPs should suffer any complement-mediated lysis. A reasonable speculation is that the direct covalent deposition of complement components (e.g., C4b and C3b) onto a percentage of the mCRPs can inhibit the functions of those mCRPs, therein creating pivotal conditions that enable complement-mediated membrane damage. High dilutions of serum also dilute complement components (and complement control proteins) resulting in a progressively lower level of complement deposition, which could be more easily controlled by the functional mCRPs on the cancer cell surface. For these reasons we chose to apply excess levels of rabbit polyclonal antibodies to $\beta_{2^{-}}$ 


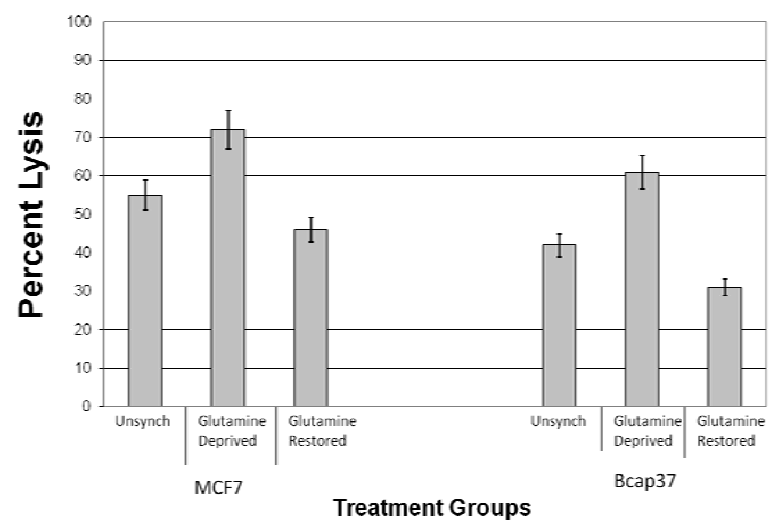

Figure 7

Percent Lysis following Glutamine Synchronization and Neutralization of CD59. Treatment groups were prepared as outlined for unsynchronized, glutamine deprived and glutamine restored populations of Bcap37 and MCF7 cells. Cells were sensitized with $20 \mu \mathrm{g}$ of rabbit polyclonal antibody to $\beta_{2}$-microglobulin and subjected to $25 \%$ fresh normal human serum (NHS) in combination with $20 \mu \mathrm{g}$ of YTH53.I rat monoclonal antibody to CD59. After 4 hours of incubation with $\mathrm{NHS}$ at $37^{\circ} \mathrm{C}$, supernatants were collected and tested for LDH activity to determine percent lysis. Please note the increase in percent lysis for both cell lines as compared to Figure 6.

microglobulin, a stable and ubiquitously expressed cell surface marker. We used $25 \%$ fresh human serum in our study of the complement-mediated killing of two different types of breast cancer cell lines, a breast adenocarcinoma cell line MCF7 [23], and a human breast medullary carcinoma cell line, Bcap37. These conditions allowed an assessment of the impact of glutamine-deprivation on cell cycle kinetics, mCRP expression and complement susceptibility in a breast cancer model.

Breast cancer cell lines subjected to a 48-hour period of glutamine-deprivation were unable to progress through mitosis, with the majority of cells trapped in G0-G1 phase. Trypan Blue exclusion verified the viability of all cells at harvesting following control and experimental treatments, including glutamine-deprivation. Restoration of glutamine to glutamine-deprived cells induced a substantial surge in cell cycle progression, with an increased proportion of cells in the G2-M phase. However, our analysis reveals that the expression of CD59 and CD55 is not dependent upon phase of the cell cycle, with G0-G1 phase cells demonstrating no significant difference in mCRP expression compared with G2-M phase cells. Yet, the restoration of glutamine to glutamine-deprived cells stimulated increased mCRP expression suggesting that CD59 and CD55 expression may be related more closely to met- abolic proliferation rather than dependent on specific phases of the cell cycle. Coincidently, the rapidly proliferating glutamine-restored breast cancer cells demonstrated increased complement resistance in association with the increased expression of CD59 and CD55. When accounting for overall cell number, the relative surface expression level of CD59 and CD55 was observed to increase appreciably in glutamine-restored breast cancer cells, which explains the associated increased complement resistance. However, other unidentified factors that impact complement susceptibility, such as membrane permeability and cytoskeleton integrity, could have also been influenced by glutamine restoration. Nonetheless, increased CD59 and CD55 expression protect breast cancer cells form antibody-directed complement activation. Particularly, the importance of CD59 was underscored in our experiments. With CD59 neutralized, unsynchronized Bcap37 and MCF7 cells demonstrated $42 \%$ and 55\% complement lysis, respectively. However, with functional CD59 activity unsynchronized Bcap37 and MCF7 cells demonstrated $26 \%$ and $37 \%$ complement lysis, respectively, indicating CD59 contributes significantly to complement resistance in unsynchronized breast cancer cells. Other studies have suggested CD59 is one of the major factors in providing host cells protection from complement activation $(29,37$, 38).

Interestingly, neutralization of CD59 in breast cancer cells deprived of glutamine resulted in maximal complementmediated damage, with lysis of $61 \%$ of Bcap 37 and $72 \%$ of MCF7 cells. The heightened complement susceptibility associated with glutamine deprivation was more than completely reversed with glutamine restoration, even if CD59 was neutralized or not. This suggests factors other than mCRPs are recruited that provide additional complement resistance to cells entering hypermetabolic states, similar to the phenomenon observed with glutamine restoration. Similar to our results, other studies have related metabolic deficiencies with increased complement susceptibility due to structurally compromised cell membranes $[22,33,34]$, which could be more easily disrupted by antibody-directed complement activation. Further studies will be needed to examine and compare each of these possibilities under the metabolic conditions induced by glutamine-deprivation.

Chemotherapeutic agents preferentially target rapidly proliferating cancer cells with the intention of inducing a tumoricidal response while minimizing collateral host damage. Many chemotherapeutic agents alter the metabolic state and cytokinetic profile in a manner not unlike glutamine-deprivation $[20,21,36]$. In future studies, the specific impact chemotherapeutic agents impose on complement susceptibility when cells are challenged with antibody-directed complement activation could provide 
valuable information in designing more effective immunotherapeutic treatments. From our studies, a theoretical advantage may be obtained from combining medical agents which increase complement susceptibility of cancer cells, either through reduced mCRP expression or altered metabolic profiles, with complement activating antibody-directed immunotherapeutic treatments.

In terms of the immunological privilege often associated with neoplasms, low levels of sustained, albeit ineffective complement deposition (e.g., at the tumor site) have been shown to propagate cancer cells that are more resistant to apoptotic signals [19] and concomitantly the surviving cells become even more resistant to complement-mediated damage $[17,18]$. This could partially explain why limited non-lethal in vivo complement activation localized to the tumor site could exacerbate the inherent pathology associated with malignancy, therein-complicating treatment efforts [18]. Malignant cancer cells also liberate soluble forms of complement mCRPs that maintain their complement-restrictive activities [4]. Thus, the evasive properties of malignant cancer cells may be attributed to an interrelated, yet not fully understood, complex of resistance mechanisms that promote cell survival and immunological privilege. Future studies will hopefully delineate the impact of specific molecular pathways associated with non-lethal complement activation and glutamine deprivation or chemotherapy on the immunological privilege and chemotherapeutic resistance commonly identified in malignant breast cancer cells.

\section{Conclusion}

Glutamine deprivation resulted in increased complement susceptibility and altered cell cycle profiles in breast cancer cells. However, glutamine restoration led to a rapid progression through the mitotic cell cycle, as well as increased CD59 and CD55 expression, which correlated with increased resistance to antibody-directed complement activation. When CD59 is neutralized, both glutamine-deprived and glutamine-restored breast cancer cells demonstrated increased complement susceptibility. These findings are important when considering the design of antibody-directed immunotherapies which attempt to selectively target cancer cells for complement-mediated killing.

\section{Methods}

\section{Cell culture media and glutamine synchronization}

The human breast adenocarcinoma cell line MCF7 [23] and the human breast medullary carcinoma cell line Bcap37 [24] were obtained from the Hollings Cancer Center at the Medical University of South Carolina. The MCF7 cells have been extensively examined for expression of $\beta_{2}$-microglobulin [23]. Adenocarcinomas are much more common than medullary mammary carcinomas, however these two very distinct cell types were employed for comparative purposes. Cell lines were customarily maintained in Delbecco's Modified Eagle Medium (DMEM) supplemented with 10\% heat-inactivated fetal bovine serum (FBS), $2 \mathrm{mM}$ L-glutamine, 50 units $/ \mathrm{ml}$ of penicillin and $50 \mu \mathrm{g} / \mathrm{ml}$ of streptomycin at $37^{\circ} \mathrm{C}$ in humidified air with 5\% carbon dioxide. All media and supplements were obtained from Fischer Scientific.

Cells were grown to confluence and then to establish treatment groups, approximately $1 \times 10^{5} \mathrm{MCF} 7$ or Bcap37 cells were inoculated onto 48-well plates. For 24 hours, treatment groups were allowed to adhere to the plate surface in DMEM media supplemented with 10\% FBS, antibiotics and $1 \mathrm{mM}$ L-glutamine at $37^{\circ} \mathrm{C}$. Next, the media was removed and cells were washed with sterile PBS. As is well-known, L-glutamine is an essential component for cell growth (Minamoto, et al, Cytotechnology. 1991;5 Suppl 2:S35-51). Treatment groups were then established based on glutamine supplementation and identified as unsynchronized, glutamine deprived or glutamine restored. "Unsynchronized" cells continued to grow uninterrupted for 48 hours in standard DMEM culture media with additional supplements as detailed above. The "glutamine deprivation" group received DMEM with 5\% FBS, antibiotics, but no glutamine for 48 hours. The conversion from 10\% FBS to 5\% FBS minimized untoward exposure of cells to extraneous L-glutamine occasionally contained in FBS preparations, but contains an adequate amount of the essential serum elements required to support basic cellular activities for 48 hours [39]. Additionally, we have observed Bcap37 and MCF7 cell lines can be continuously grown in DMEM supplemented with 5\% FBS and $2 \mathrm{mM}$ L-glutamine. The "glutamine restored" cells were treated identical to the "glutamine deprived" cells for 48 hours, but then received DMEM with 10\% FBS and $2 \mathrm{mM}$ L-glutamine for 8 hours before harvesting. The 48-hour period of glutamine-deprivation synchronized cells in a quiescent G0-G1 phase of the cell cycle, allowing comparison of glutamine-deprived cells with unsynchronized cells and cells treated with glutamine deprivation followed by glutamine restoration.

\section{Complement-activating antibodies and normal human serum}

Approximately $1 \times 10^{5} \mathrm{MCF} 7$ or Bcap37 cells were inoculated onto 48-well plates with each well supplemented with DMEM media with 10\% fetal bovine serum (FBS), antibiotics, and glutamine. To induce complement activation, cells were sensitized with either $10 \mu \mathrm{g}(20 \mu \mathrm{g} / \mathrm{ml})$ or $20 \mu \mathrm{g}(40 \mu \mathrm{g} / \mathrm{ml})$ of rabbit polyclonal antibody to human $\beta_{2}$-microglobulin (anti- $\beta 2 \mathrm{M}$, Accurate Inc.), a ubiquitously expressed cell surface marker associated with Major Histocompatibility Complex-1 antigen [25]. When indicated, antibody-mediated neutralization of CD59 func- 
tion was achieved using YTH53.1 (Serotec), a rat monoclonal antibody to CD59 proven to neutralize its complement-inhibitory function $(37,38)$. The YTH53.1 has demonstrated high specificity for human CD59 and effectively neutralizes it complement inhibition properties, thus negative antibody controls testing the specificity of YTH53.1 were not necessary $(37,38,42,43)$. Specified amounts of $0,5,10$, or $20 \mu \mathrm{g}$ representing $0 \mu \mathrm{g} / \mathrm{ml}, 10 \mu \mathrm{g} /$ $\mathrm{ml}, 20 \mu \mathrm{g} / \mathrm{ml}$ and $40 \mu \mathrm{g} / \mathrm{ml}$ absolute concentrations of YTH53.1 were added in combination with an optimized level $(20 \mu \mathrm{g})$ of rabbit polyclonal anti-human $\beta_{2^{-}}$ microglobulin (anti- $\beta$ 2M). Following antibody treatments, cells were incubated with normal human serum as a source of complement. Normal human serum (NHS) was obtained from venous blood donated with consent by healthy volunteers (blood was allowed to clot for one hour at room temperature and for two hours on ice-water, then centrifuged, aliquoted and stored at minus $80^{\circ} \mathrm{C}$ ). Specified dilutions of NHS were made in sterile isotonic barbital buffered saline, $\mathrm{BBS}^{++}\left(0.15 \mathrm{mM} \mathrm{Ca}^{++}\right.$and $1.0 \mathrm{mM}$ $\left.\mathrm{Mg}^{++}\right)$, pH 7.3 and placed in frozen storage. Aliquots were thawed in warm water bath for 30 minutes prior to use.

\section{Lactate dehydrogenase-release-assay to detect complement-mediated damage}

Lactate dehydrogenase (LDH) is an intracellular enzyme released into the extracellular environment when a cell is damaged by lytic perforations induced in the cell membrane via complement activation. Triplicate LDH assays were performed with 48-well plates (Fischer Scientific) with each sample containing approximately $1 \times 10^{5} \mathrm{MCF} 7$ or Bcap37 cells. Incubations with NHS were for $30 \mathrm{~min}$ at $37^{\circ} \mathrm{C}$; then $0.4 \mathrm{mM}$ PMSF was added to prevent degradation of LDH by endogenously released intracellular proteases. Subsequently, these cells were incubated with the human serum for 4 additional hours at $37^{\circ} \mathrm{C}$ to elicit a maximum cellular response to complement activation. Application of PMSF simultaneously blocks new complement activation, yet we have observed that with additional time the cells with damaged membranes expand [26] and release LDH. Background detection of LDH was obtained with $1 \times 10^{5}$ cells incubated without antibodies in the presence of NHS, and was subtracted from experimental values. The LDH-assay solution (Sigma Diagnostics) was prepared according to the standard commercial protocol provided by Sigma Diagnostics. After each treatment group was incubated with NHS and specified antibody combinations, $50 \mu \mathrm{l}$ samples were taken from the supernatant and added to $1 \mathrm{ml}$ of the LDH-assay solution, mixed thoroughly and read at $340 \mathrm{~nm}$. Total (100\%) lysis was obtained by treating the cells with $1 \%$ Triton-X100 detergent. The percentage of lysis was determined by dividing the experimental LDH activity by the total lysis $\mathrm{LDH}$ value. At the conclusion of each LDH assay, Trypan Blue exclusion was used to corroborate the percentage of cells with membrane damage (percent of living cells) as compared to the LDH assay. An ANOVA statistical analysis revealed no significant variation between the commercial LDH detection assay and the Trypan Blue exclusion, revealing the $\mathrm{LDH}$ detection method to be a reliable method in the determination of complement-mediated cell membrane damage. In addition, Trypan Blue exclusion was used to verify viability of all cells at harvesting following control and experimental treatments.

\section{Flow cytometry: determination of cell cycle distribution and $m C R P$ expression}

Unsynchronized or glutamine synchronized treatment groups were prepared for flow cytometry analysis. Cells were harvested with cold Versene (Gibco) to obtain a single-suspension of cells and then centrifuged at $1000 \mathrm{rpm}$ for $5 \mathrm{~min}$ at $27^{\circ} \mathrm{C}$. Pellets were re-suspended in $1 \%$ bovine serum albumin (BSA) blocking solution and centrifuged at $1000 \mathrm{rpm}(200 \mathrm{~g})$ for $5 \mathrm{~min}$ at $27^{\circ} \mathrm{C}$. Pellets were re-suspended in serum-free DMEM and aliquots of approximately $1 \times 10^{6}$ cells were probed with FITClabeled monoclonal antibody to either CD55 $(2 \mu \mathrm{g} / \mathrm{ml}$ mouse IgG1 BRIC-216, Serotec Inc.) or to CD59 (2 $\mathrm{gg} / \mathrm{ml}$ mouse IgG2a, MEM43, Serotec Inc.). Isotype (negative) controls were non-specific FITC-labeled mouse immunoglobulins, IgG2a and IgG1. Concomitantly, cells were fixed with ice-cold 95\% ethanol ( $\mathrm{pH} 7.4$ ). The cells were resuspended in $100 \mu \mathrm{l}$ of $0.5 \mathrm{mg} / \mathrm{ml}$ propidium iodide and $0.1 \mathrm{mg} / \mathrm{ml}$ ribonuclease A in $0.15 \mathrm{M}$ PBS. The treated cells were filtered through a $35-\mu \mathrm{m}$ cell-strainer and analyzed using a FACSCalibur ${ }^{\mathrm{TM}}$ (Becton Dickinson) flow cytometer with a $488 \mathrm{~nm}$ argon-ion laser for excitation. Light emission as a function of propidium iodide intercalated within DNA (to identify the cell-cycle) was detected using a $585 \mathrm{~nm}$ bandpass filter and the data analyzed using ModFit LT ${ }^{\mathrm{TM}}$ (Verity) software. Populations of cells were identified in either G0-G1 or G2-M based on channel values dictated by the amount of propidium iodide present in each cell, representing different stages of cell cycle. All Diploid cells were identified, then separated according to channel values based on propidium iodide fluorescence at $585 \mathrm{~nm}$. Cells identified as G0-G1 had channel values ranging approximately from 25 to 75 . Cells identified as G2-M had channel values ranging approximately from 140 to 175 . Gating was performed manually after identification of G0-1 and G2-M populations to further determine CD59 and CD55 Mean Fluorescent Intensity (MFI) among these distinct populations of cells at different stages in the cell cycle. The acquisition threshold was set at 5,000 cells using the ModFit LT software, which allowed 5,000 diploid cells to be identified in the G0-G1 and G2-M channel ranges, and then these cells were further evaluated for MFI of CD59 and CD55 expression. Using the negative background isotype controls (non-specific FITC-labeled mouse immunoglobulins, IgG2a 
and $\operatorname{IgG}$ ), no binding of the negative control antibodies to the cell surface was identified among MCF7 and Bcap37 cells. The positive fluorescent antibody control (fluorescently tagged anti- $\beta_{2}$-microglobulin) revealed uniform and near-ubiquitous expression of $\beta_{2}$-microglobulin among all MCF7 and Bcap37 cells irrespective of cell cycle phase or glutamine synchronization. Data was analyzed using CellQuest $^{\mathrm{TM}}$ (Becton Dickinson) software. Instrument performance was routinely monitored using DNA QC Particles and Calibrite ${ }^{\mathrm{TM}}$ beads (Becton Dickinson).

\section{Abbreviations}

MCF7, adenocarcinoma breast cancer cell line; Bcap37, medullary breast cancer cell line; mCRPs, membrane complement regulatory proteins; CD55, Decay Accelerating Factor; CD59, protectin; LDH, lactate dehydrogenase; $\beta 2 \mathrm{M}, \beta_{2}$-microglobulin; FBS, fetal bovine serum.

\section{Competing interests}

The author(s) declare that they have no competing interests.

\section{Authors' contributions}

BSE carried out the experiments. MKBZ supplied the interpretation of the results and with RJB wrote the manuscript. All authors read and approved the manuscript.

\section{Acknowledgements}

This work was supported in part by the Department of Defense Congressionally Mandated Breast Cancer Research Program, grant number DAMDI7-02-I-0564 and in part by NIH R2I AI069957.

\section{References}

I. Donev RM, Cole DS, Sivasankar B, Hughes TR, Morgan B: p53 regulates cellular resistance to complement lysis through enhanced expression of CD59. Cancer Res 2006, 66:245I-2458.

2. Hakulinen J, Meri S: Complement-mediated killing of microtumors in vitro. Am J Pathol 1998, I 53:845-855.

3. Bjørge L, Matre R: Down-regulation of CD59, protectin. expression on human colorectal adenocarcinoma cell lines by levamisole. Scand I Immunol I995, 42:5 I 2-5 I6.

4. Spendlove I, Ramage JM, Bradley R, Harris C, Durrant LG: Complement decay accelerating factor DAF/CD55 in cancer. Cancer Immunol Immunother 2006, 55:987-995.

5. Bjørge L, Hakulinen J, Vintermyr OK, Jarva H, Jensen TS, Iversen OE, Meri S: Ascitic complement system in ovarian cancer. $\mathrm{Br} J \mathrm{Can}$ cer 2005, 92:895-905.

6. Bjørge L, Stoiber H, Dierich MP, Meri S: Minimal residual disease in ovarian cancer as a target for complement-mediated $\mathbf{m A b}$ immunotherapy. Scand J Immunol 2006, 63:355-364

7. Loberg RD, Day L, Dunn R, Kalikin LM, Pienta KJ: Inhibition of decay-accelerating factor CD55 attenuates prostate cancer growth and survival in vivo. Neoplasia 2006, 8:69-78.

8. Livingston PO, Hood C, Krug LM, Warren N, Kris MG, Brezicka T, Ragupathi G: Selection of GM2., fucosyl GMI., globo $H$ and polysialic acid as targets on small cell lung cancers for antibody mediated immunotherapy. Cancer Immunol Immunother 2005, 54: I0I8-1025.

9. Rushmere N, Knowiden JM, Gee JM, Harper ME, Robertson JF, Morgan BP, Nicholson RI: Analysis of the level of mRNA expression of the membrane regulators of complement. CD59, CD55 and CD46 in breast cancer. Int J Cancer 2004, I 08:930-936.

10. Chen $\mathrm{CH}$, Boackle RJ: A newly discovered function for $\mathbf{C l}$ inhibitor, removal of the entire $\mathrm{Clqr}_{2} \mathrm{~s}_{2}$ complex from immobi- lized human IgG subclasses. Clin Immunol Immunopathol I998, 87:68-74.

II. Chen $\mathrm{CH}$, Lam CF, Boackle RJ: Cl inhibitor removes the entire $\mathrm{CI} \mathrm{qr}_{2} \mathrm{~s}_{2}$ complex from anti-CIQ monoclonal antibodies with low binding affinities. Immunology 1998, 95:648-654.

12. Boackle RJ, Nguyen QL, Leite RS, Yang X, Vesely J: ComplementCoated Antibody-Transfer, CCAT; Serum IgAI Antibodies Intercept and Transport C4 and C3 Fragments and Preserve IgG I Deployment, PGD. Molecular Immunology 2006, 43:236-245.

13. Donin N, Jurianz K, Ziporen L, Schultz S, Kirschfink M, Fishelson Z: Complement resistance of human carcinoma cells depends on membrane regulatory proteins, protein kinases and sialic acid. Clin Exp Immunol 2003, I 3 I :254-263.

14. Gelderman KA, Tomlinson S, Ross GD, Gorter A: Complement function in mAb-mediated cancer immunotherapy. Trends Immunol 2004, 25: I 58-164.

I5. Cudrici C, Niculescu F, Jensen T, Zafranskaia E, Fosbrink M, Rus V, Shin ML, Rus H: C5b-9 terminal complex protects oligodendrocytes from apoptotic cell death by inhibiting caspase-8 processing and up-regulating FLIP. J Immunol 2006, I 76:3173-3180.

16. Zwaka TP, Torzewski J, Hoeflich A, Dejosez M, Kaiser S, Hombach V, Jehle PM: The terminal complement complex inhibits apoptosis in vascular smooth muscle cells by activating an autocrine IGF-I loop. FASEB J 2003, I 7: I346-I348.

17. Reiter Y, Ciobotariu A, Fishelson Z: Sublytic complement attack protects tumor cells from lytic doses of antibody and complement. Eur J Immunol 1992, 22: I207-1213.

18. Marchbank KJ, van den Berg CW, Morgan BP: Mechanisms of com plement resistance induced by non-lethal complement attack and by growth arrest. Immunology 1997, 90:647-653.

19. Fosbrink M, Niculescu F, Rus H: The role of C5b-9 terminal complement complex in activation of the cell cycle and transcription. Immunol Res 2005, 31:37-46.

20. Leibson PJ, Schreiber H, Loken MR, Panem S, Rowley DA: Timedependent resistance or susceptibility of tumor cells to cytotoxic antibody after exposure to a chemotherapeutic agent. Proc Natl Acad Sci USA 1978, 75:6202-6206.

21. Leibson PJ, Loken MR, Shapiro SJ, Schreiber H: Direct determination of the influence of the cell cycle on the survival of tumor cells exposed to cytotoxic antibodies. Cancer Res 1980, 40:56-60.

22. Ohanian SH, Yamazaki M, Schlager SI, Faibisch M: Cell growthdependent variation in the sensitivity of human and mouse tumor cells to complement-mediated killing. Cancer Res I983, 43:491-495.

23. Ogretmen B, McCauley MD, Safa AR: Molecular mechanisms of loss of beta 2-microglobulin expression in drug-resistant breast cancer sublines and its involvement in drug resistance. Biochemistry 1998, 37:11679-11691.

24. Sui M, Dziadyk JM, Zhu X, Fan W: Cell cycle-dependent antagonistic interactions between paclitaxel and gamma-radiation in combination therapy. Clin Cancer Res 2004, 1 0:4848-4857.

25. Gobbi G, Mirandola P, Micheloni C, Solenghi E, Sponzilli I, Artico M, Soda G, Zanelli G, Pelusi G, Fiorini T, Cocco L, Vitale M: Expression of HLA class I antigen and proteasome subunits LMP-2 and LMP-I 0 in primary vs. metastatic breast carcinoma lesions. Int J Oncol 2004, 25:1625-1629.

26. Weaver JL, Boackle RJ, Ades EW: Rapid kinetic method for detection of complement mediated damage to nucleated cells. J Immunol Methods 1980, 4I:257-266.

27. Stein GS, Stein JG, Lian JB, Last TJ, Owen TA, McCabe L: Cell Biology 1998. A laboratory Handbook. Volume I. Second edition. Edited by: Celis JE. Academic Press, San Diego

28. Jurianz K, Ziegler S, Garcia-Schuler H, Kraus S, Bohana-Kashtan O, Fishelson Z, Kirschfink M: Complement resistance of tumor cells: basal and induced mechanisms. Mol Immunol 1999 , 36:929-939

29. Madjd Z, Pinder SE, Paish C, Ellis IO, Carmichael J, Durrant LG: Loss of CD59 expression in breast tumours correlates with poor survival. J Pathol 2003, 200:633-639.

30. Madjd Z, Durrant LG, Bradley R, Spendlove I, Ellis IO, Pinder SE: Loss of CD55 is associated with aggressive breast tumors. Clin Cancer Res 2004, 10:2797-2803. 
31. Huang Y, Qiao F, Abagyan R, Hazard S, Tomlinson S: Defining the CD59-C9 binding interaction. I Biol Chem 2006, 28I:27398-27404.

32. Andoh A, Shimada M, Araki Y, Fujiyama Y, Bamba T: Sodium butyrate enhances complement-mediated cell injury via down-regulation of decay-accelerating factor expression in colonic cancer cells. Cancer Immunol Immunother 2002, 50:663-672.

33. Schlager SI, Ohanian SH: Role of membrane lipids in the immunological killing of tumor cells, I. Target cell lipids. Lipids 1983, 18:475-482.

34. Pilzer D, Fishelson Z: Mortalin/GRP75 promotes release of membrane vesicles from immune attacked cells and protection from complement-mediated lysis. Int Immunol 2005, I7:1239-1248.

35. Hurtubise A, Momparler RL: Effect of histone deacetylase inhibitor LAQ824 on antineoplastic action of 5-Aza-2'-deoxycytidine, decitabine. on human breast carcinoma cells. Cancer Chemother Pharmacol 2006, 58:618-625.

36. Shapiro SJ, Leibson PJ, Loken MR, Schreiber H: Changes in susceptibility to cytotoxic antibody among tumor cells surviving exposure to chemotherapeutic agents. Cancer Res 1982, 42:2622-2627.

37. Jarvis GA, Li J, Hakulinen J, Brady KA, Nordling S, Dahiya R, Meri S: Expression and function of the complement membrane attack complex inhibitor protectin (CD59) in human prostate cancer. Int J Cancer 7 I (6): I049-55. I 997 Jun II

38. Brasoveanu LI, Altomonte M, Fonsatti E, Colizzi F, Coral S, Nicotra MR, Cattarossi I, Cattelan A, Natali PG, Maio M: Levels of cell membrane CD59 regulate the extent of complement-mediated lysis of human melanoma cells. Lab Invest 1996 74(I):33-42

39. Wasa M, Bode BP, Abcouwer SF, Collins CL, Tanabe KK, Souba WW: Glutamine as a regulator of DNA and protein biosynthesis in human solid tumor cell lines. Ann Surg 1996, 224(2):189-97.

40. Fishelson Z, Donin N, Zell S, Schultz S, Kirschfink M: Obstacles to cancer immunotherapy: expression of membrane complement regulatory proteins (mCRPs) in tumors. Mol Immunol 2003, 40(2-4): 109-23.

41. Minamoto Y, Ogawa K, Abe H, lochi Y, Mitsugi K: Development of a serum-free and heat-sterilizable medium and continuous high-density cell culture. Cytotechnology 1991, 5(Suppl 2):S35-5I.

42. Coral S, Fonsatti E, Sigalotti L, De Nardo C, Visintin A, Nardi G, Colizzi F, Colombo MP, Romano G, Altomonte M, Maio M: Overexpression of protectin (CD59) down-modulates the susceptibility of human melanoma cells to homologous complement. J Cell Physiol 2000, 185(3):3 17-23.

43. Hakulinen J, Meri S: Complement-mediated killing of microtumors in vitro. Am J Pathol 1998, I 53(3):845-55.

Publish with Bio Med Central and every scientist can read your work free of charge

"BioMed Central will be the most significant development for disseminating the results of biomedical research in our lifetime. "

Sir Paul Nurse, Cancer Research UK

Your research papers will be:

- available free of charge to the entire biomedical community

- peer reviewed and published immediately upon acceptance

- cited in PubMed and archived on PubMed Central

- yours - you keep the copyright

Submit your manuscript here:

http://www.biomedcentral.com/info/publishing_adv.asp
BioMedcentral 